\title{
Telesurgery System for Intravascular Neurosurgery
}

\author{
Mitsutaka Tanimoto*1, Fumihito Arai*1, Toshio Fukuda*2 \\ Kouichi Itoigawa*3, Masashi Hashimoto*3 \\ Ikuo Takahashi*4 and Makoto Negoro*5 \\ *1 Dept. of Micro System Engineering, Graduate School of Engineering, \\ Nagoya University \\ Furo-cho, Chikusa-ku, Nagoya, 464-8603, JAPAN \\ *2 Center for Cooperative Research in Advanced Science \& Technology, \\ Nagoya University \\ *3 TOKAI RIKA Co., LTD. \\ *4 Anjyo Kousei Hospital \\ *5 Dept. of Neurosurgery, Nagoya University
}

\begin{abstract}
The intravascular neurosurgery is one of the most successful minimally invasive surgery. However, the difficulty to operate a catheter inside the cranial blood vessels because of the complexity and narrowness of blood vessels checks increasing the number of well-skilled doctors. Moreover the Xray camera, which is used during an operation, causes the X-ray exposure to a doctor. One of the solution to these problems is telesurgery system

A novel telesurgery system for intravascular neurosurgery is described in this paper. This system has three main components; 1) A micro force sensor which can be installed in the catheter tip, and it measures a contact force between the catheter tip and a blood vessel wall. 2) A catheter teleoperation system which enables a surgeon to perform a medical operation to a patient from a distant place. 3) A force display strategy to improve operability and safety during an operation.

An in-vitro teleoperation experiment in the intravascular operating room is also performed and the effectiveness of the telesurgery system is confirmed.
\end{abstract}

\section{Introduction}

In recent years, minimum invasive surgery is getting greater attention in the medical field. This technique allows us to reduce physical pain and aftereffects for patients. Here we focus on the intravascular neurosurgery as an example. A catheter is one of the medical tools frequently used in the intravascular surgeries. A catheter is inserted into blood vessels from the groin and navigated far deep into cranial blood vessels from outside of a body through blood vessels.

To operate a catheter inside the cranial blood vessels is very difficult because of the complexity and narrowness of blood vessels. Moreover, this difficulty causes not only an extension of operating time, but also the fatigue of the operators and patients. So there is a problem that there are not so many well-skilled doctors who can operate a catheter appropriately as compared with the number of patients who want to be treated by the intravascular surgery. Moreover, these doctors are always exposed to X- 
ray radiation whenever they perform an operation. A solution for these problems is a telesurgery system [1]-[4]. So we have studied about telesurgery system for several years, and confirm the possibility of the telesurgery system [1][5].

To perform telesurgery using teleoperation technology, correct and easy information display to understand the situation of slave side (patient side) is very important.

In catheter operation, doctors use only X-ray image as visual information source when they operate a catheter in the patient's blood vessels. The X-ray video is monochrome and it is difficult to understand the condition of catheter and blood vessels especially when the contrast is not clear. So doctors regard their tactile information as important when theyoperate a catheter.

In this paper, we focus on the force display method as an approach of our study. We develop a novel catheter teleoperation system, which is suitable to the catheter operation, and it can realize a fine force display. We also show a new control strategy to improve the performance of safety and operability by two force display methods. One is a multiple force sensor information display method, which displays several forces that are measured by several force sensors on one master device. The other one is a variable impedance characterization of master joystick, which changes the impedance parameter of the master joystick according to the situation of the medical operation. After that a result of the experiment to evaluate these force display method is shown. Finally we show the result of an in-vitro telesurgery experiment in which a doctor is not exposed to the X-ray while the catheter operation.

\section{Policy of a System Design}

\subsection{Problems in the Catheter Teleoperation}

Doctors have to maneuver the catheter tip from the inserting hole where is one meter away from the catheter tip. Because the catheter is a very flexible thin tube, control input cannot be transferred to the tip of catheter correctly. Moreover, we can not measure the position of the catheter tip correctly because there is no space to install any position sensor in the catheter tip, and there is also no useful position sensing method from the outside of the body. This means that we cannot use any method, which needs accurate position information.

Doctors regard the tactile information as very important to estimate the situation between a catheter and blood vessels. Especially they want to know the situation between the catheter tip and blood vessel wall because the penetration of a blood vessel wall is the most dangerous situation in accidents.

So we consider that the tactile information is the most important information source for the catheter operation.

\subsection{Policy of a System Design}

In our system the position information is provided to the doctors by the video. We do not assist the doctors in taking the position information. Of course we do understand the effectiveness of the visual assistant method such as the navigation map, computer graphic overlaying. But in this paper, we focus on the force display approach to take 
up the problems, which were mentioned in the previous paragraph. We need the following functions;

1. Fine force reproduction on the master joystick

2. A mechanism which can modify the impedance parameter of the master joystick to change the operability

3. Precise position tracking of slave device

To realize these functions, we employ the force reflection type master slave system as the basic structure.

In the normal force reflection type master slave system, the mechanical impedance of the master arm can not be removed, therefore, this causes bad feeling of the force display. However, to use the impedance control, we can change the mechanical impedance of the master arm and reduce the influence to the force display.

Doctors give a position command to a catheter during a catheter operation. So slave unit must follow the master position as accurate as possible. The position tracking gain should be enough high to make a fine position tracking servo. But if the impedance of the environment is too high, the system become more unstable as the position servo gain become higher. In the case of the catheter operation, the environment is a blood vessel, which has very low impedance, therefore, the system don't become unstable even if we give high position tracking gain to the slave device.

We also take it into account that we should reduce the mechanical impedance of the master joystick when we design the system.

\section{Hardware of a Catheter Teleoperation System}

We designed a 2 DOF (forward \& backward, rotation) master slave system. 2 DOF is necessary and sufficient for the catheter operation. The master joystick has very small mechanical impedance. The slave device has 2-axis force sensor, which measures the contact force between blood vessels and a catheter. There is a micro force sensor in the "tip" of a catheter, which measures the contact force between the catheter tip and the blood vessel wall. The detail is shown in the next section.

\subsection{Structure of a Master Joystick}

The master joystick is shown in Fig. 1, and motion mechanism is shown in Fig. 2. The first joint is a primastic joint and this joint is connected to the forward and backward motion of a catheter. The second joint is a revolute joint and this joint corresponds to rotating motion of a catheter. Each joint is connected to a motor with a wire mechanism. There is no gear between the motor and the joint to reduce the influence of the friction and backlash. Each joint has a optical rotary encoder, and the resolution of the position measurement is 1.6 micro meter per pulse, 0.023 degree per pulse, respectively.

\subsection{Structure of a Slave Device}

The slave device is shown in Fig. 3, and the mechanism is shown in Fig. 4. There is a inner unit in an outer shell. The catheter is put between a pair of rollers. A motor is 
connected to the rollers, and a catheter is driven forward and backward by the rollers. The outer shell rounds to generate the rotating motion of the catheter.

The inner unit is hung from the outer shell with thin beams. There is a strain gauge on the thin beam. This is a force sensor, which measures the pushing component of the contact force between a catheter and a blood vessel. The two rollers are connected by thin beams. There is also a strain gauge on the thin beam. This force sensor measures the rotating component of the contact force. The force measurement mechanism is shown in Fig. 5a and Fig. 5b.

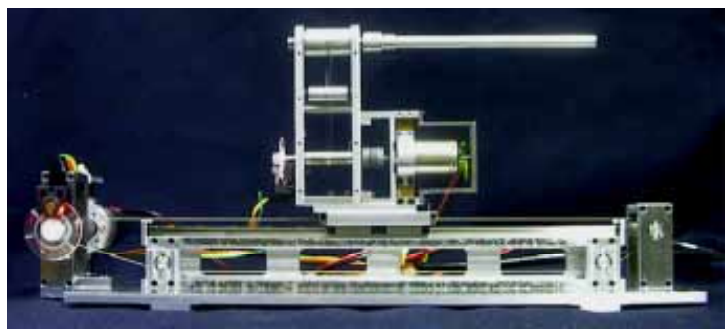

Fig. 1 Master joystick

This joystick has $2 \mathrm{DOF}$

(forward \& backward, rotation).

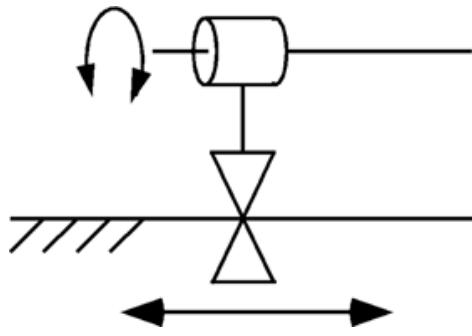

Fig. 2 Mechanism of the master joystick

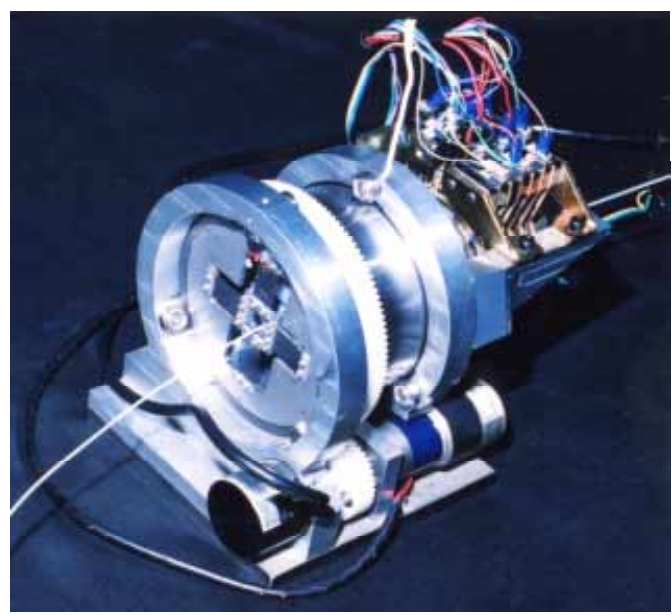

Fig. 3 Slave device; The slave device has 2 DOF (forward \& backward, rotation) and there is a strain gauge force sensor for each axis. This device was first designed in 1994, and has been improved for several years. 


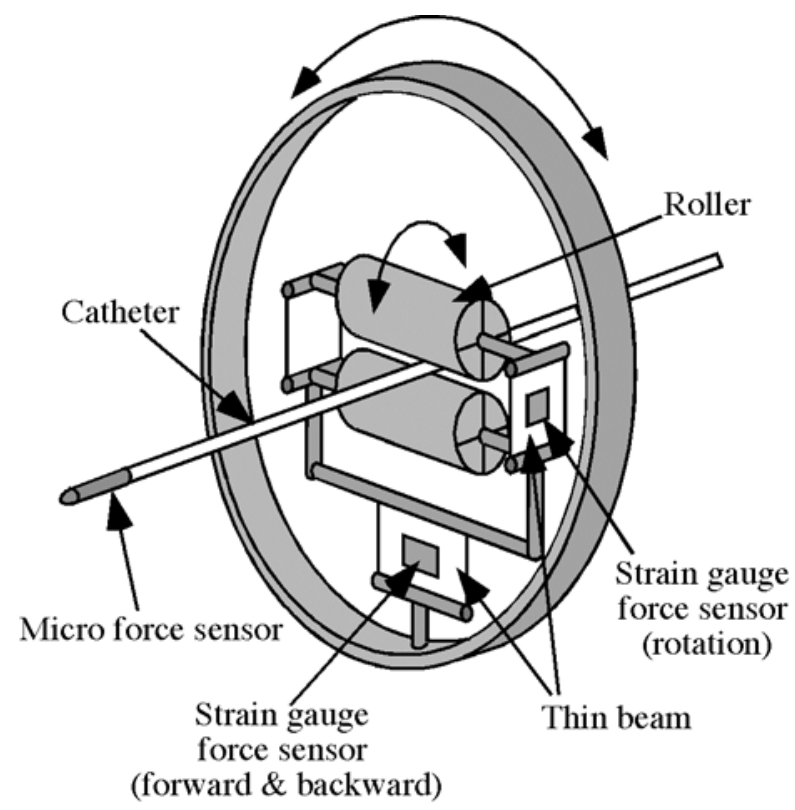

Fig. 4 Mechanism of the slave device

\subsection{Micro Force Sensor[6]}

A micro force sensor is installed in the tip of a catheter. We have developed this small force sensor for several years and we show the latest version of the micro force sensor here. The diameter and the length is $1.2 \mathrm{~mm}, 5 \mathrm{~mm}$, respectively. The sensor is shown in Fig. 6. Doctors can measure the contact force between the catheter tip and the blood vessel well. The resolution is less than $0.5 \mathrm{mN}$, and frequency response is fine up to $2 \mathrm{kHz}$.

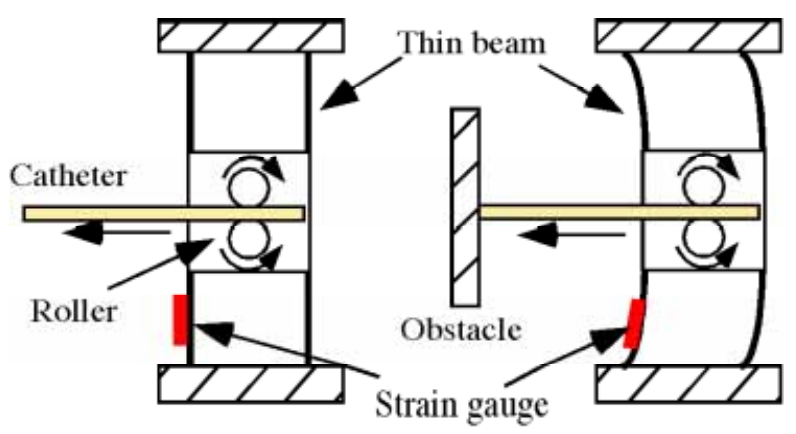

Fig. 5a Mechanism of force measurement (forward \& backward) 
When the catheter makes contact with the blood vessel wall, the catheter movement is disturbed. But the roller continues to push the catheter. So the pushing force is measured by the strain gauge force sensor.

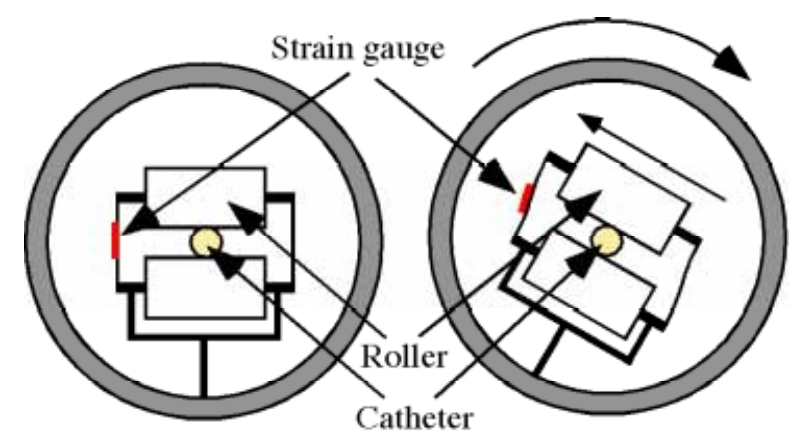

Fig. 5b Mechanism of force measurement (rotation)

There is some frictional force between the catheter and the blood vessel wall while the catheter operation. Therefore, even if the slave device becomes turn round, the catheter stays in the same position. At that time, the upper roller will move to the side direction, and this force is measured by the strain gauge on the thin beam.

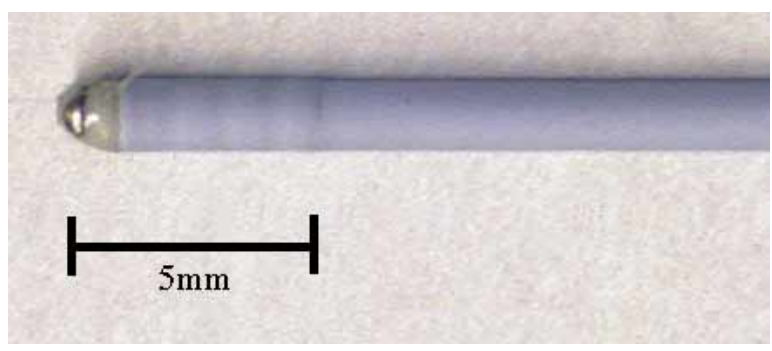

Fig. 6 Micro force sensor

Diameter $1.2 \mathrm{~mm}$, Length $5 \mathrm{~mm}$, Resolution $0.5 \mathrm{mN}$

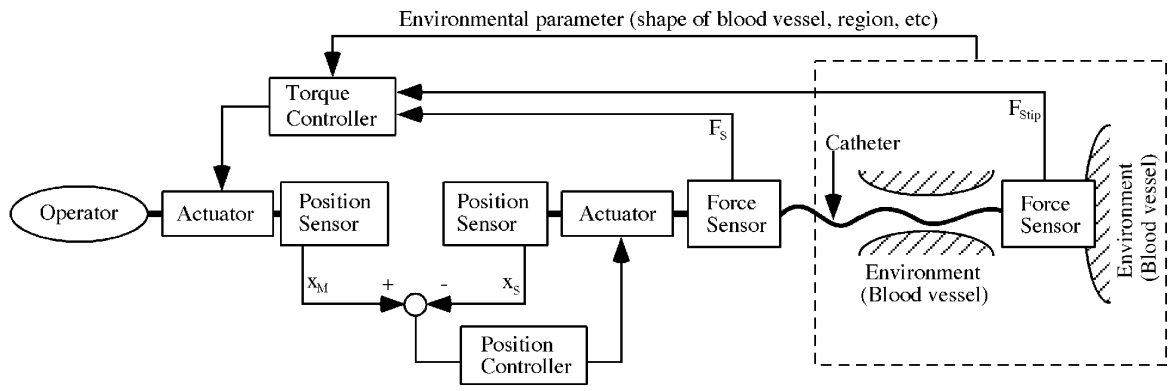

Fig. 7 Catheter teleoperation system 


\section{Force Display Method for the Catheter Teleoperation System}

As we wrote before, we use the force reflection type master slave system as our basic structure. Our system is shown in Fig. 7. We also adopt the impedance control to change the impedance parameter of the master joystick. The target impedance parameter is changed according to the situation of the slave side. We also display 2 forces, which are measured by the strain gauge force sensor at slave device and by the micro force sensor at the tip of a catheter.

\subsection{Multiple Force Sensor Information Display Method}

Here we focus on the force display of the forward \& backward motion. Force information is measured by two force sensors (strain gauge force sensor, micro force sensor). In the usual catheter operation, the force information is transmitted to the operator through the catheter so the operator can't distinguish between those two forces. But we can display these two forces independently because we can measure these two kinds of force respectively.

The force, which is measured by the strain gauge force sensor at the slave device, is always measured whenever the catheter is in the blood vessels. This force is changed according to the movement of the catheter. On the other hand, the force, which is measured by the micro force sensor, does not appear until the catheter tip and the blood vessel wall contact. At that time an operator will feel the contact force as if the catheter contacts to a hard wall. To select an appropriate magnifying coefficient of the contact force at the catheter tip, the operator can distinguish these two forces even if the two forces are displayed on the same master joystick. The formulation of the multiple force sensor information display is written as follows;

$$
\begin{aligned}
& F_{\text {sensor }}=K_{F S} F_{S}+K_{F S t i p} F_{S t i p} \\
& K_{F S}, K_{\text {FStip }} \neq 0
\end{aligned}
$$

where, $\mathrm{F}_{\text {sensor }}$ is a total force, $\mathrm{F}_{\mathrm{S}}, \mathrm{F}_{\text {Stip }}$ is a force which is measured by the strain gauge and the micro force sensor, respectively. $\mathrm{K}_{\mathrm{FS}}, \mathrm{K}_{\mathrm{FStip}}$ is magnifying coefficient of each force.

\subsection{Variable Impedance Characterization (VIC)}

In the intravascular surgery, it is difficult to recover from a mistake when a serious situation for a patient occurs. The system must have a function to reduce operator's mistakes. We realize this function by changing the impedance of the master joystick. By selecting a large master joystick impedance, the operator will not be able to move the joystick very fast. In this way the system can reduce the possibility to injure the blood vessel wall by the operator. We also use the impedance changing mechanism to improve the operability of maneuvering the catheter teleoperation system. Generally, there is an appropriate impedance size for each task, and a human naturally changes their arm or finger impedance parameter to match the task to do. But in the 
teleoperation system, a mechanical system disturbs an operator to get the appropriate impedance for the task. So we make the system to realize an appropriate impedance for the operator. We call this impedance changing mechanism as Variable Impedance Characterization (VIC). The VIC changes the impedance of the master arm dynamically by reflecting the situation of the slave side. The basic concept is written as;

$$
\begin{gathered}
F_{v}=M_{v} \ddot{x}_{M}+C_{v} \dot{x}_{M} \\
M_{v}=M_{v}\left(x_{S}, D, S, \text { etc. }\right) \\
C_{V}=C_{v}\left(x_{S}, D, S, \text { etc. }\right)
\end{gathered}
$$

where, $x_{M}$ is a position of master arm, $F_{V}$ is a virtual resistant force against the operator. $\mathrm{M}_{\mathrm{V}}, \mathrm{C}_{\mathrm{V}}$ are virtual mass and virtual coefficient of viscosity, respectively. $\mathrm{x}_{\mathrm{S}}$ is a parameter of the catheter position. In the catheter operation, the two degrees of freedom are needed (motion along axial direction, and rotating motion). Basically the map of the blood vessels is expressed by a parameter of inserted length. To give an appropriate function to $\mathrm{x}_{\mathrm{S}}$, we can express the information of the blood vessel structure such as a position of a branch approximately. $\mathrm{D}$ is a diameter of the blood vessel where the tip of a catheter is placed. $\mathrm{D}$ will be measured in a prior checkup. If the catheter tip is in a thick blood vessel, an operator can move the catheter quickly, without increasing the risk of injuring blood vessels. But if $\mathrm{D}$ is getting smaller, an operator should not move the catheter so fast. So $M_{V}$ and $C_{V}$ will be inverse proportion to the diameter with a proper function. $\mathrm{S}$ is a parameter which represents the shape of the blood vessels such as a joint angle of a branch or curvature of the vessel.

These concepts are shown in Fig. 8. The virtual resistant force will become strong as the blood vessel is smaller and more complex. To summarize the force display method for the master joystick, it is described as;

$$
\begin{aligned}
\mathrm{F}_{\mathrm{M}} & =-\left(\mathrm{F}_{\text {sensor }}+\mathrm{F}_{\mathrm{V}}\right) \\
& =-\left(\mathrm{K}_{\mathrm{FS}} \mathrm{f}_{\mathrm{S}}+\mathrm{K}_{\text {FStip }} \mathrm{f}_{\text {Stip }}+\mathrm{M}_{\mathrm{V}} \ddot{x}_{\mathrm{M}}+\mathrm{C}_{\mathrm{V}} \dot{\mathrm{x}}_{\mathrm{M}}\right)
\end{aligned}
$$
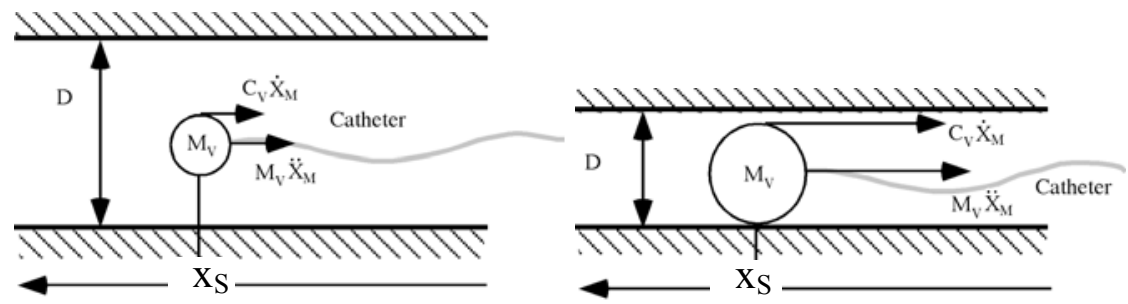

Fig. 8 (a) Virtual resistant force for master arm when the catheter tip is in a thick vessel
Fig. 8 (b) Virtual resistant force when the catheter tip is in a thin vessel or near a branch 


\section{In-Vitro Telesurgery Experiment without the X-Ray Exposure}

An in-vitro telesurgery experiment is performed in the intravascular operating room. An experimental configuration is shown in Fig. 11. The master joystick is placed in the next room of the intravascular operating room. A doctor controls a catheter or a guidewire 5 meter away from a patient (blood vessel model made of soft plastic tube) and the X-ray camera. A doctor can see the X-ray image on the monitor but he can't see the blood vessel model directly. Force information, which is measured by 2 force sensors (micro force sensor in the tip of a catheter and the strain gauge force sensor at the slave device), is displayed on the master joystick. Therefore a doctor can feel force information. A scene of the experiment is shown in Fig. 12-14. Figure 12 shows a doctor's operation. He didn't wear a X-ray protector whole while the operation. Figure 13 is a scene of the patient side. A catheter is inserted into the blood vessel model as same as the doctor's direct operation. Figure 14 is a $\mathrm{X}$ ray image which is displayed to the operator. The effectiveness of the catheter teleoperation system has been confirmed through this experiment.

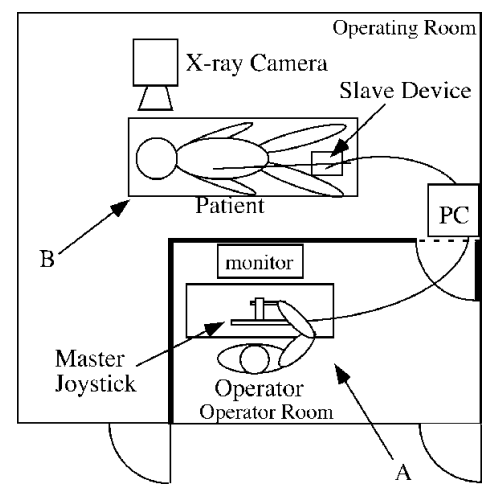

Fig. 11 Experimental Configuration

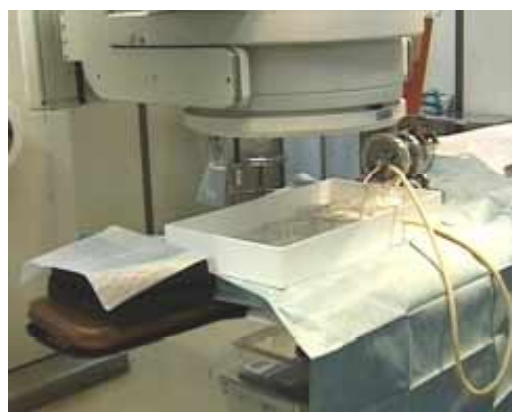

Fig. 13 Patient side

(from view point B in Fig. 11)

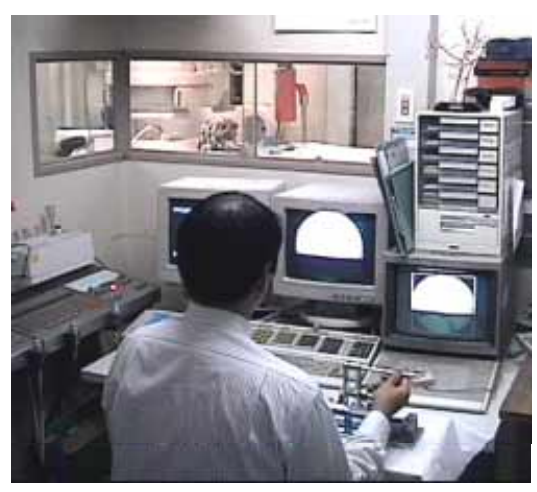

Fig. 12 Operator side (from view point A in Fig. 11)

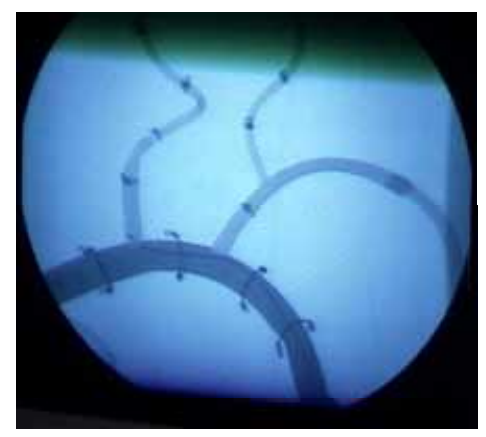

Fig. $14 \mathrm{X}$-ray image which is displayed to a operator 


\section{Toward Practical Use}

\section{Easy Sterilization}

The part, which contacts with the catheter, should be disposable to realize an easy, cheap but perfect sterilization system. The parts which contact to the catheter are rollers and the guide sheath which will be attached in front and behind of the rollers to support the catheter. By exchanging these parts, we can easily keep the contact point sterilized. We also have to insulate the motor, the rotary encoder and the force sensor from the water or sterilize gas to be able to reuse these parts.

\section{Safety}

Automatic emergency stop mechanism should be added to the control law. One of the most dangerous situation is to penetrate the blood vessel wall. The micro force sensor will be able to detect a sign of danger before actual penetration. Some warning sign to the operator should also be needed at the sometime.

\section{Conclusion}

In this paper, we have shown a new catheter teleoperation system, and we also proposed a new force display method which improves the safety and operability during a catheter teleoperation. We described two methods. One is a multiple force sensor information display (two forces are; 1. total frictional force between the catheter and blood vessels, 2. contact force between the catheter tip and blood vessels) on the master joystick. The other one is a Variable Impedance Characterization (VIC), which changes the impedance of the master joystick dynamically to reflect the situation of a catheter and blood vessels. Finally we have shown an in-vitro telesurgery experiment and confirmed the effectiveness of our system.

\section{Acknowledgement}

This research was partially supported by the Ministry of Education, Science, Sports and Culture, Grant-in-Aid for Scientific Research (No.10003371).

\section{References}

[1] F. Arai, et al, "Multimedia Tele-surgery Using High Speed Optical Fiber Network and Its Application to Intravascular Neurosurgery", Proc. IEEE Int'l Conf. on Robotics and Automation ICRA'96, Vol.1, pp.878-883, 1996.4

[2] M. Mitsuishi, Y. Iizuka, H. Nakanishi, et al., "Remote operation of a micro surgical System", Proc. IEEE International Conference on Robotics and Automation, pp.10131019, 1998

[3] S. E. Salcudean, et al., "Performance Measurement in Scaled Teleoperation for Microsurgery”, CVRMed-MRCAS'97, Grenoble, France, pp.789-798, 1997.

[4] J.W. Hill, "Telepresence Surgery Demonstration System", Proc. 1994 IEEE Int'l Conf. on Robotics and Automation, Vol. 3, pp. 2302-2307, 1994 
[5] M.Tanimoto, et al., "Augmentation of Safety in Teleoperation System for Intravascular Neurosurgery”, IEEE Int'l Conf. on Robotics and Automation, pp. 2890 -2895, 1998

[6] M.Tanimoto, et al., "Micro Force Sensor for Intravascular Neurosurgery", IEEE Int'1 Conf. on Robotics and Automation, Vol. 2, pp. 1561-1566, 1997

[7] K.S. Kwon, et al., "A Robot with Improved Adcolute Positioning Accuracy for CT Guided Stereotactic Surgery", IEEE Trans. on Biomedical Engineering, pp. 153-161, February 1988

[8] S.E. Salcudean and J. Yen, "Towards a Force-reflecting Motion-Scaling System for Microsurgery”, Proc. 1994 IEEE Int'l Conf. on Robotics and Automation, Vol. 3, pp. 2296-2301, 1994

[9] K.Kaneko, et al., "Impedance Shaping based on Force Feedback Bilateral Control in Macro-Micro Teleoperation System”, IEEE Int'l Conf. on Robotics and Automation, Vol. 1, pp. 710-717, 1997 